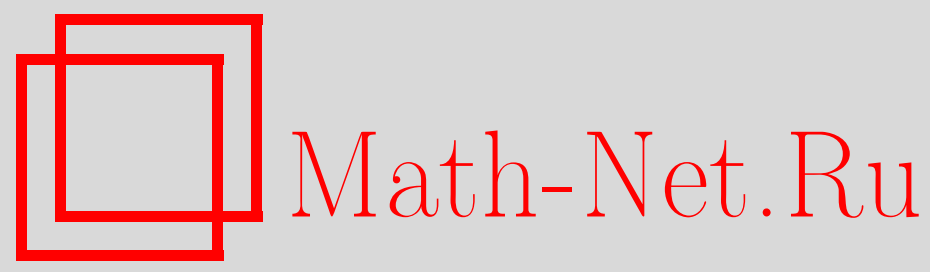

В. Н. Безверхний, И. В. Добрынина, О неразрешимости проблемы сопряженности подгрупп в группе крашеных кос $R_{5}$, Матем. заметки, 1999, том 65, выпуск 1, 15-22

DOI: https://doi.org/10.4213/mzm1023

Использование Общероссийского математического портала Math-Net.Ru подразумевает, что вы прочитали и согласны с пользовательским соглашением http://www.mathnet.ru/rus/agreement

Параметры загрузки:

IP: 3.85 .73 .92

26 апреля 2023 г., 15:05:52

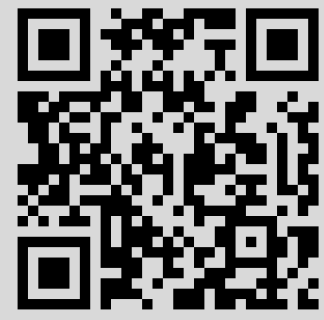




\title{
О НЕРАЗРЕШИМОСТИ ПРОБЛЕМЫ СОПРЯЖЕННОСТИ ПОДГРУПП В ГРУППЕ КРАШЕНЫХ КОС $R_{5}$
}

\section{В.Н. Безверхний, И. В. Добрынина}

\begin{abstract}
Основная цель работы - доказательство неразрешимости проблемы сопряженности подгрупп в $R_{5}$.

Библиограффия: 8 названий.
\end{abstract}

Группа кос $B_{n+1}$ задается образуюшими $\sigma_{1}, \ldots, \sigma_{n}$ и определяюшими соотношениями

$\sigma_{i} \sigma_{i+1} \sigma_{i}=\sigma_{i+1} \sigma_{i} \sigma_{i+1}, \quad i=1, \ldots, n-1, \quad \sigma_{i} \sigma_{j}=\sigma_{j} \sigma_{i}, \quad i, j=1, \ldots, n, \quad|i-j|>1$.

Известно, что существует гомоморфизм группы $B_{n+1}$ в группу подстановок $S_{n+1}$, при котором образующие $\sigma_{i}$ отображаются в транспозиции $(i, i+1), i=1, \ldots, n$. Коса, реализующая единичную подстановку, называется крашеной. Через $R_{n+1}$ обозначается подгруппа крашеных кос группы $B_{n+1}$, через $s_{i, j}(1 \leqslant i<j \leqslant n+1)$ - коса $\sigma_{j-1} \sigma_{j-2} \cdots \sigma_{i+1} \sigma_{i}^{2} \sigma_{i+1}^{-1} \cdots \sigma_{j-2}^{-1} \sigma_{j-1}^{-1}$ группы $B_{n+1} \cdot$ В [1] В. Бурау доказал, что элементы $s_{i, j}(1 \leqslant i<j \leqslant n+1)$ порождают группу $R_{n+1}$. Через $U_{j}(j=1, \ldots, n)$ обозначается подгруппа группы крашеных кос $R_{n+1}$, порожденная элементами $s_{1, j+1}, s_{2, j+1}$, $\ldots, s_{j, j+1}$. В [2] Э. Артин доказал, что $U_{j}-$ свободная группа, порожденная свободньми образующими $s_{1, j+1}, s_{2, j+1}, \ldots, s_{j, j+1}$, и что всякая крашеная коса из $R_{n+1}$ однозначно представима в виде произведения $F_{1} F_{2} \cdots F_{n}$, где $F_{j} \in U_{j}(j=1, \ldots, n)$.

Пусть $U_{m-1}-$ свободная группа с образующими $s_{1, m}, s_{2, m}, \ldots, s_{m-1, m}$. Для любых $r, p(1 \leqslant r<p<m)$, любого $i(1<i<m)$ и любого $\xi= \pm 1$ справедливы соотношения [3]

$$
s_{r, p}^{-\xi} s_{i, m} s_{r, p}^{\xi}=\left\{\begin{array}{l}
s_{i, m}, \text { если } r<p<i<m \text { или } i<r<p<m, \\
\left(s_{r, m} s_{p, m}\right)^{\xi} s_{i, m}\left(s_{r, m} s_{p, m}\right)^{-\xi}, \\
\quad \text { если } r<i=p<m \text { или } r=i<p<m, \\
\left(s_{r, p} s_{p, m}\right)^{\xi}\left(s_{p, m} s_{r, m}\right)^{-\xi} s_{i, m}\left(s_{p, m} s_{r, m}\right)^{\xi}\left(s_{r, m} s_{p, m}\right)^{-\xi}, \\
\text { если } r<i<p<m .
\end{array}\right.
$$

В [4] доказана неразрешимость проблемы сопряженности подгрупп для групп $R_{n+1}$ $(n \geqslant 5)$. Цель данной работы - доказательство неразрешимости проблемы сопряженности подгрупп для группы $R_{5}$. В работе используется следуюшая 


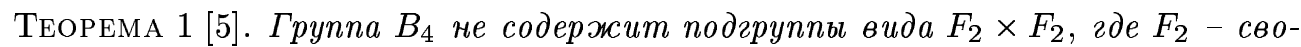
бодная группа ранга 2.

Обозначим через $d$ слово $\sigma_{4} \sigma_{3} \sigma_{2} \sigma_{1}^{2} \sigma_{2} \sigma_{3} \sigma_{4}$, а через $\tau$ переписьвающий процесс слов из $R_{5}$ в образующих $s_{i, j}$. Тогда $\tau(d)=s_{1,5} s_{2,5} s_{3,5} s_{4,5}$. Рассмотрим в $B_{5}$ подгруппы $\Pi=\left\langle\sigma_{1}^{4}, \sigma_{2}^{4}\right\rangle \times\left\langle\sigma_{4}^{2}, d\right\rangle, H_{1}=\left\langle\sigma_{1}^{4} \sigma_{4}^{2}, \sigma_{2}^{4} d, R_{1}\left(\sigma_{1}^{4}, \sigma_{2}^{4}\right), \ldots, R_{n}\left(\sigma_{1}^{4}, \sigma_{2}^{4}\right)\right\rangle, H_{2}=\left\langle W, H_{1}\right\rangle$, где $W \in \Pi$ и $R_{i}\left(\sigma_{1}^{4}, \sigma_{2}^{4}\right)(i=1, \ldots, n)$ - слова в образуюших $\sigma_{1}^{4}, \sigma_{2}^{4}$.

ЛЕМма 1. Подгруппы $\left\langle\sigma_{1}^{4}, \sigma_{2}^{4}\right\rangle,\left\langle\sigma_{4}^{2}, d\right\rangle$ группы $B_{5}$ являются свободными.

ДокАЗАтЕльство. Рассмотрим гомоморфизм $\varphi: B_{3}=\left\langle\sigma_{1}, \sigma_{2} ; \sigma_{1} \sigma_{2} \sigma_{1}=\sigma_{2} \sigma_{1} \sigma_{2}\right\rangle \rightarrow$ $G=\left\langle x, y ; x^{3}, y^{2}\right\rangle$, отображающий $\sigma_{1}$ в $x^{2} y, \sigma_{2}$ в $y x^{2}$. Заметим, что в произведениях $\varphi\left(\sigma_{i}^{4 \xi_{1}}\right) \varphi\left(\sigma_{j}^{4 \xi_{2}}\right)\left(i, j=1,2\right.$ и $i \neq j$ при $\left.\xi_{1}=-\xi_{2}\right)$ максимум сокрашается по одному слогу из $\varphi\left(\sigma_{i}^{4 \xi_{1}}\right)$ и $\varphi\left(\sigma_{j}^{4 \xi_{2}}\right)$ и по одному - объединяется.

Пусть $\left\langle\sigma_{1}^{4}, \sigma_{2}^{4}\right\rangle$ не является свободной. Тогда несократимое слово, записанное в образующих $\sigma_{1}^{4}, \sigma_{2}^{4}$, равно 1 в группе $B_{3}$. Поэтому образ этого слова равен 1 и в $G$. Но из сделанного вьше замечания следует, что это невозможно.

Группа $\left\langle\sigma_{4}^{2}, d\right\rangle$ является подгруппой $U_{4}$, причем $\sigma_{4}^{2}$ и $d$ могут быть включены в систему свободных образующих $U_{4}$, поэтому $\left\langle\sigma_{4}^{2}, d\right\rangle$ свободна.

Tеорема 2. Eсли $z^{-1} H_{1} z=H_{2}$, где $z \in R_{5}$, mo cyществует $z^{*}=L_{1}\left(\sigma_{1}^{4}, \sigma_{2}^{4}\right) \times$ $L_{2}\left(\sigma_{4}^{2}, d\right)$ maкое, что $z^{*-1} H_{1} z^{*}=H_{2}$ в $R_{5}$.

ДокАЗАТЕЛЬСТво. Представим $z$ в виде $z=F_{1} F_{2} F_{3} F_{4}$, где $F_{j} \in U_{j}$.

Из равенства $z^{-1} H_{1} z=H_{2}$ следует вьполнение равенств системы:

$$
\begin{gathered}
F_{4}^{-1} F_{3}^{-1} F_{2}^{-1} F_{1}^{-1} \sigma_{1}^{4} \sigma_{4}^{2} F_{1} F_{2} F_{3} F_{4}=\varphi_{1}\left(\sigma_{1}^{4}, \sigma_{2}^{4}\right) \varphi_{2}\left(\sigma_{4}^{2}, d\right), \\
F_{4}^{-1} F_{3}^{-1} F_{2}^{-1} F_{1}^{-1} \sigma_{2}^{4} d F_{1} F_{2} F_{3} F_{4}=\varphi_{3}\left(\sigma_{1}^{4}, \sigma_{2}^{4}\right) \varphi_{4}\left(\sigma_{4}^{2}, d\right), \\
F_{4}^{-1} F_{3}^{-1} F_{2}^{-1} F_{1}^{-1} R_{i}\left(\sigma_{1}^{4}, \sigma_{2}^{4}\right) F_{1} F_{2} F_{3} F_{4}=\varphi_{5, i}\left(\sigma_{1}^{4}, \sigma_{2}^{4}\right) \varphi_{6, i}\left(\sigma_{4}^{2}, d\right), \quad i=1, \ldots, n .
\end{gathered}
$$

Заметим, что в $(2),(3)$ все слова $F_{j}(j=1, \ldots, 4)$ записаны в образующих $\sigma_{i}(i=1, \ldots, 4)$.

Лемма 2. В $R_{5}$ подсистема (2) системы (2), (3) әквивалентна системе

$$
\begin{gathered}
F_{2}^{-1} F_{1}^{-1} \sigma_{1}^{4} F_{1} F_{2}=\varphi_{1}\left(\sigma_{1}^{4}, \sigma_{2}^{4}\right), \quad F_{2}^{-1} F_{1}^{-1} \sigma_{2}^{4} F_{1} F_{2}=\varphi_{3}\left(\sigma_{1}^{4}, \sigma_{2}^{4}\right) \\
\left(F_{2}^{-1} F_{1}^{-1} \sigma_{1}^{-4} F_{1} F_{2}\right) F_{3}^{-1}\left(F_{2}^{-1} F_{1}^{-1} \sigma_{1}^{4} F_{1} F_{2}\right) F_{3}=1 \\
\left(F_{2}^{-1} F_{1}^{-1} \sigma_{2}^{-4} F_{1} F_{2}\right) F_{3}^{-1}\left(F_{2}^{-1} F_{1}^{-1} \sigma_{2}^{4} F_{1} F_{2}\right) F_{3}=1 \\
\left(F_{2}^{-1} F_{1}^{-1} \sigma_{1}^{-4} F_{1} F_{2}\right) F_{4}^{-1}\left(F_{2}^{-1} F_{1}^{-1} \sigma_{1}^{4} F_{1} F_{2}\right) F_{3}^{-1} \sigma_{4}^{2} F_{3} F_{4}=\varphi_{2}\left(\sigma_{4}^{2}, d\right) \\
\left(F_{2}^{-1} F_{1}^{-1} \sigma_{2}^{-4} F_{1} F_{2}\right) F_{4}^{-1}\left(F_{2}^{-1} F_{1}^{-1} \sigma_{2}^{4} F_{1} F_{2}\right) F_{3}^{-1} d F_{3} F_{4}=\varphi_{4}\left(\sigma_{4}^{2}, d\right) .
\end{gathered}
$$

ДокАЗАТЕЛьство. Преобразуем систему (2) следующим образом:

$$
\begin{aligned}
& \left(F_{2}^{-1} F_{1}^{-1} \sigma_{1}^{4} F_{1} F_{2}\right)\left(F_{2}^{-1} F_{1}^{-1} \sigma_{1}^{-4} F_{1} F_{2}\right) F_{3}^{-1}\left(F_{2}^{-1} F_{1}^{-1} \sigma_{1}^{4} F_{1} F_{2}\right) F_{3} \\
& \quad \times\left(F_{3}^{-1} F_{2}^{-1} F_{1}^{-1} \sigma_{1}^{-4} F_{1} F_{2} F_{3}\right) F_{4}^{-1}\left(F_{3}^{-1} F_{2}^{-1} F_{1}^{-1} \sigma_{1}^{4} F_{1} F_{2} F_{3}\right) F_{3}^{-1} \sigma_{4}^{2} F_{3} F_{4} \\
& \quad=\varphi_{1}\left(\sigma_{1}^{4}, \sigma_{2}^{4}\right) \varphi_{2}\left(\sigma_{4}^{2}, d\right), \\
& \left(F_{2}^{-1} F_{1}^{-1} \sigma_{2}^{4} F_{1} F_{2}\right)\left(F_{2}^{-1} F_{1}^{-1} \sigma_{2}^{-4} F_{1} F_{2}\right) F_{3}^{-1}\left(F_{2}^{-1} F_{1}^{-1} \sigma_{2}^{4} F_{1} F_{2}\right) F_{3} \\
& \quad \times\left(F_{3}^{-1} F_{2}^{-1} F_{1}^{-1} \sigma_{2}^{-4} F_{1} F_{2} F_{3}\right) F_{4}^{-1}\left(F_{3}^{-1} F_{2}^{-1} F_{1}^{-1} \sigma_{2}^{4} F_{1} F_{2} F_{3}\right) F_{3}^{-1} d F_{3} F_{4} \\
& \quad=\varphi_{3}\left(\sigma_{1}^{4}, \sigma_{2}^{4}\right) \varphi_{4}\left(\sigma_{4}^{2}, d\right) .
\end{aligned}
$$


В силу однозначности представления всякого элемента $y \in R_{n+1}$ в виде $y=y_{1} y_{2} \cdots y_{n}$, где $y_{j} \in U_{j}$, из (7) получаем системы (4), (5) и систему

$$
\begin{gathered}
\left(F_{3}^{-1} F_{2}^{-1} F_{1}^{-1} \sigma_{1}^{-4} F_{1} F_{2} F_{3}\right) F_{4}^{-1}\left(F_{3}^{-1} F_{2}^{-1} F_{1}^{-1} \sigma_{1}^{4} F_{1} F_{2} F_{3}\right) F_{3}^{-1} \sigma_{4}^{2} F_{3} F_{4}=\varphi_{2}\left(\sigma_{4}^{2}, d\right), \\
\left(F_{3}^{-1} F_{2}^{-1} F_{1}^{-1} \sigma_{2}^{-4} F_{1} F_{2} F_{3}\right) F_{4}^{-1}\left(F_{3}^{-1} F_{2}^{-1} F_{1}^{-1} \sigma_{2}^{4} F_{1} F_{2} F_{3}\right) F_{3}^{-1} d F_{3} F_{4}=\varphi_{4}\left(\sigma_{4}^{2}, d\right) .
\end{gathered}
$$

Используя (5), преобразуем систему (8) к виду (6). Обратное утверждение очевидно.

Фундаментальньй элемент

$$
\sigma_{1} \sigma_{2} \sigma_{3} \cdots \sigma_{n-1} \sigma_{n} \sigma_{1} \sigma_{2} \sigma_{3} \cdots \sigma_{n-2} \sigma_{n-1} \cdots \sigma_{1} \sigma_{2} \sigma_{3} \sigma_{1} \sigma_{2} \sigma_{1}
$$

группы $B_{n+1}$ обозначается через $\Delta[5]$ и $\Delta^{2}$ лежит в центре $B_{n+1}$. Графическое равенство слов будем обозначать через “т”.

Лемма 3. Пусть $H_{1}, H_{2}$ - две конечно порожденные подгруппы $и з\left\langle\sigma_{1}^{4}, \sigma_{2}^{4}\right\rangle$ ини одна из них не сопря⿻ена с подгруппами вида $\left\langle\sigma_{j}^{4 t}\right\rangle(j=1,2, t \in \mathbb{Z})$. Если при этом существует $z^{*} \in B_{3}$ такое, что $z^{*} H_{1} z^{*-1}=H_{2}$ в $B_{3}$, mо $z^{*}=\Delta^{k} S\left(\sigma_{1}^{4}, \sigma_{2}^{4}\right)$,

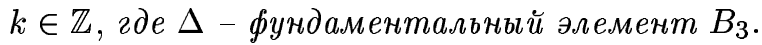

ДокАЗАТЕЛЬСтво. Пусть $B_{3}=\left\langle\sigma_{1}, \sigma_{2} ; \sigma_{1} \sigma_{2} \sigma_{1}=\sigma_{2} \sigma_{1} \sigma_{2}\right\rangle$. Рассмотрим гомоморфизм $\varphi: B_{3} \rightarrow G=\left\langle x, y ; x^{3}, y^{2}\right\rangle$, переводящий $\sigma_{1}$ в $x^{2} y, \sigma_{2}$ в $y x^{2}$. Тогда

$$
\varphi\left(\sigma_{1}^{4}\right)=\left(x^{2} y\right)^{4} ; \quad \varphi\left(\sigma_{2}^{4}\right)=\left(y x^{2}\right)^{4} .
$$

Обозначим через $z$ слово $\varphi\left(z^{*}\right)$.

В подгруппе $\varphi\left(H_{1}\right)$ выберем циклически несократимое в образующих $\varphi\left(\sigma_{1}^{4}\right), \varphi\left(\sigma_{2}^{4}\right)$ слово $T$. Этого всегда можно добиться, сопрягая $\varphi\left(H_{1}\right), \varphi\left(H_{2}\right)$ элементами подгруппы $\left\langle\varphi\left(\sigma_{1}^{4}\right), \varphi\left(\sigma_{2}^{4}\right)\right\rangle$.

Исходя из условия леммы, считаем, что $T$ не есть слово $\sigma_{j}^{4 t}(j=1,2, t \in \mathbb{Z})$. Возведем $T$ в степень $m$, где $m \in \mathbb{N}$ и $m>1$, и пусть $W \mp T^{m}$.

Из равенства $z \varphi\left(H_{1}\right) z^{-1}=\varphi\left(H_{2}\right)$ следует, что $z$ можно выбрать минимальным в двойном смежном классе $\varphi\left(H_{1}\right) z^{-1}\left\langle\varphi\left(\sigma_{1}^{4}\right), \varphi\left(\sigma_{2}^{4}\right)\right\rangle$, при необходимости сопрягая обе части данного равенства словами из $\left\langle\sigma_{1}^{4}, \sigma_{2}^{4}\right\rangle$. Из равенства $z W z^{-1}=V$, где $V \in \varphi\left(H_{2}\right)$, получаем, что $z W z^{-1}$ записьвается через $\varphi\left(\sigma_{1}^{4}\right), \varphi\left(\sigma_{2}^{4}\right)$.

Возможны случаи:

1) $W=\left(x^{2} y\right)^{4 \xi} W^{\prime}\left(y x^{2}\right)^{4 \eta}, \xi= \pm 1, \eta= \pm 1$;

2) $W$ 프 $\left(y x^{2}\right)^{4 \xi} W^{\prime}\left(y x^{2}\right)^{4 \xi}, \xi= \pm 1$

3) $W=\left(y x^{2}\right)^{4 \xi} W^{\prime}\left(x^{2} y\right)^{4 \eta}, \xi= \pm 1, \eta= \pm 1$;

4) $W \mp\left(x^{2} y\right)^{4 \xi} W^{\prime}\left(x^{2} y\right)^{4 \xi}, \xi= \pm 1$.

Случай 1 ) при $\xi=\eta=-1$ сводится к случаю 1$)$ при $\xi=\eta=1$ сопряжением подгруп $\varphi\left(H_{1}\right), \varphi\left(H_{2}\right)$ элементом $y$ и заменой $W$ словом $W^{-1}$, так как сопряжение элементом $y=\varphi(\Delta)$ в группе $\varphi\left(B_{3}\right)$ является инволютивным автоморфизмом.

Случай 2) при $\xi=-1$ сводится к случаю 2$)$ при $\xi=1$ заменой $W$ словом $W^{-1}$.

Случай 3 ) сводится к случаю 1$)$ сопряжением подгрупп $\varphi\left(H_{1}\right), \varphi\left(H_{2}\right)$ элементом $y$. Тем же способом случай 4) сводится к случаю 2).

Заметим, как и в доказательстве леммы 1 , что в произведениях $\varphi\left(\sigma_{i}^{4 \xi_{1}}\right) \varphi\left(\sigma_{j}^{4 \xi_{2}}\right)(i, j=$ 1,2 и $i \neq j$ при $\left.\xi_{1}=-\xi_{2}\right)$ максимум сокращается по одному слогу из $\varphi\left(\sigma_{i}^{4 \xi_{1}}\right)$ и $\varphi\left(\sigma_{j}^{4 \xi_{2}}\right)$ и по одному - объединяется. 
Будем считать, что в произведении $z W^{\xi} z^{-1}, \xi= \pm 1$, сокрашения происходят в подслове $W^{\xi} z^{-1}$.

Рассмотрим случай 1$)$ при $\xi=\eta=1$, т.е. $W \mp\left(x^{2} y\right)^{4} W^{\prime}\left(y x^{2}\right)^{4}$.

При этом возможно:

a) $z=\approx z^{\prime} y x$

б) $z \mp z^{\prime} y x^{2}$.

Рассмотрим случай а). Имеем $z W z^{-1} \mp z^{\prime} y x\left(x^{2} y\right)^{4} W^{\prime}\left(y x^{2}\right)^{4} x^{-1} y^{-1} z^{\prime-1}$. Возьмем обратное:

$$
z W^{-1} z^{-1}=z^{\prime} y x\left(x^{-2} y\right)^{4} W^{\prime}\left(y x^{-2}\right)^{4} x^{-1} y z^{\prime-1}=z^{\prime} y x^{2} y x y x y x W^{\prime-1}\left(y x^{-2}\right)^{3} z^{\prime-1} .
$$

В силу выбора $z$ и $W$ следует, что в данном равенстве сокращения не затрагивают $z^{\prime}$. Так как $z W^{-1} z^{-1}$ переписьвается в образуюших $\varphi\left(\sigma_{1}^{4}\right), \varphi\left(\sigma_{2}^{4}\right)$, в силу минимальности $z$ и сделанного вьше замечания слоговая длина $z^{\prime}$ не превосходит 4 и $z^{\prime}$ является подсловом $\varphi\left(\sigma_{i}^{2 \xi}\right)(i=1,2, \xi= \pm 1)$. Таким образом, $z^{\prime}$ может быть равным $1, x, x^{2}, y x, y x^{2}$, $x^{2} y x^{2}, y x y x, y x^{2} y x^{2}, x y x, x^{2} y x, y x^{2} y x$.

Если $z^{\prime}$ есть $1, x, x^{2}, y x, y x^{2}$, то легко убедиться, что слово $z W z^{-1}$ нельзя переписать в образующих $\varphi\left(\sigma_{1}^{4}\right), \varphi\left(\sigma_{2}^{4}\right)$.

Если $z^{\prime}$ равно $x^{2} y x^{2}, y x y x, y x^{2} y x^{2}, x y x, x^{2} y x, y x^{2} y x$, то $z$ не является минимальньм в классе $\varphi\left(H_{1}\right) z^{-1}\left\langle\varphi\left(\sigma_{1}^{4}\right) \varphi\left(\sigma_{2}^{4}\right)\right\rangle$.

Аналогично рассматривается случай б).

В случае 1) при $\xi=-1, \eta=1$ и $\xi=1, \eta=-1$ и в случае 2) при $\xi=1$ в слове $z W z^{-1}$ либо $z$, либо $z^{-1}$ не затрагивается сокращениями, поэтому слоговая длина $z$ не превосходит 4 и $z$, как и выше, есть подслово $\varphi\left(\sigma_{i}^{2 \xi}\right)(i=1,2, \xi= \pm 1)$.

Проверка таких $z$ не дает положительных результатов.

Если в произведении $z W z^{-1}$ ни $z$, ни $z^{-1}$ не вступают с $W$ в сокращения, то в силу равенства $z W z^{-1}=V$, где $V \in \varphi\left(H_{2}\right), z$ является подсловом $\varphi\left(\sigma_{i}^{2 \xi}\right)(i=1,2, \xi= \pm 1)$. Рассматривая эти подслова, получаем, что $z$ может быть либо 1 , либо $y$, где $y=\varphi(\Delta)$.

Возвращаясь в группу $B_{3}$, получаем, что $z^{*}=\Delta^{k} S\left(\sigma_{1}^{4}, \sigma_{2}^{4}\right)$.

СлЕдСТВИЕ. Пусть $H_{1}, H_{2}$ - две конечно порохденные подгруппы $u з\left\langle\sigma_{1}^{4}, \sigma_{2}^{4}\right\rangle, u$ ни одна из них не сопряжена с подгруппами вида $\left\langle\sigma_{j}^{4 t}\right\rangle(j=1,2, t \in \mathbb{Z})$. Если при этом существует $z^{*} \in R_{3}$ такое, что $z^{*} H_{1} z^{*-1}=H_{2}$ в $R_{3}$, mо $z^{*}=\Delta^{2 k} S\left(\sigma_{1}^{4}, \sigma_{2}^{4}\right)$,

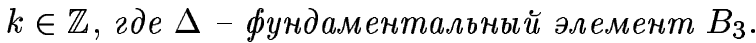

Лемма 4. Если $z^{\prime}=F_{1} F_{2}\left(F_{i} \in U_{i}, i=1,2\right)$ удовлетворяет системе (4), то существует коса $z^{\prime \prime}=L_{1}\left(\sigma_{1}^{4}, \sigma_{2}^{4}\right)$, являющаяся решением (4).

Доказательство непосредственно следует из следствия леммы 3.

ЛЕмма 5. Если $F_{3} \in U_{3}$ является решением системы $(5)$, то $\tau\left(F_{3}\right)=\left(s_{1,4}, s_{2,4}\right.$, $\left.s_{3,4}\right)^{k}, k \in \mathbb{Z}$.

ДокАЗАтЕльство. Очевидно, коса $F_{3}=\left(\sigma_{3} \sigma_{2} \sigma_{1}^{2} \sigma_{2} \sigma_{3}\right)^{k}, k \in \mathbb{Z}$, является решением системы (5), причем $\tau\left(F_{3}\right)=\left(s_{1,4} s_{2,4} s_{3,4}\right)^{k}$. Если существует коса $X \in U_{3}$, являющаяся решением (5) и не равная степени элемента $\sigma_{3} \sigma_{2} \sigma_{1}^{2} \sigma_{2} \sigma_{3}$, то в силу принадлежности $X$ и $F_{3}$ свободной группе $U_{3}$ и в силу равенства $(5)$ в $B_{4}$ содержится прямое произведение двух свободных групп ранга $2\left\langle F_{2}^{-1} F_{1}^{-1} \sigma_{1}^{4} F_{1} F_{2}, F_{2}^{-1} F_{1}^{-1} \sigma_{2}^{4} F_{1} F_{2}\right\rangle \times\left\langle X, F_{3}\right\rangle$, что противоречит теореме 1 . 
ЛЕмма 6. В $R_{5}$ имеет место соотношение $\tau(d)\left(s_{1,4} s_{2,4} s_{3,4}\right)=\left(s_{1,4} s_{2,4} s_{3,4}\right) \tau(d)$.

ДокАЗАТЕЛЬСТво. Непосредственно используя равенства (1) получаем доказательство данного утверждения.

Лемма 7. В $R_{5}$ имеет место соотношение

$$
\left(s_{1,4} s_{2,4} s_{3,4}\right)^{-k} s_{4,5}\left(s_{1,4} s_{2,4} s_{3,4}\right)^{k}=\tau^{k}(d) s_{4,5} \tau^{-k}(d), \quad k \in \mathbb{Z}
$$

Доказательство проводится по индукции, используя соотношения (1) и лемму 6.

ЛЕмма 8. Пусть имеет место система (2) и соответственно выполнена әквивалентная ей система (4)-(6). Тогда если $F_{4} \in U_{4}$ удовлетворяет системе (6), mo $F_{4}=F_{4}\left(\sigma_{4}^{2}, d\right)$.

ДокАЗАТЕЛЬСТво. Обозначим через $\left|F_{4}\right|$ длину слова $F_{4} \in U_{4}$ в свободных образующих $s_{4,5}, s_{3,5}, s_{2,5}, \tau(d)$. Возьмем $k \in \mathbb{N}$ такое, что $k$ намного больше $2\left|F_{4}\right|$. Возведем равенства $(2)$ в $k$-ю степень. Получим

$$
\begin{gathered}
F_{4}^{-1} F_{3}^{-1} F_{2}^{-1} F_{1}^{-1} \sigma_{1}^{4 k} \sigma_{4}^{2 k} F_{1} F_{2} F_{3} F_{4}=\varphi_{1}^{k}\left(\sigma_{1}^{4}, \sigma_{2}^{4}\right) \varphi_{2}^{k}\left(\sigma_{4}^{2}, d\right), \\
F_{4}^{-1} F_{3}^{-1} F_{2}^{-1} F_{1}^{-1} \sigma_{2}^{4 k} d^{k} F_{1} F_{2} F_{3} F_{4}=\varphi_{3}^{k}\left(\sigma_{1}^{4}, \sigma_{2}^{4}\right) \varphi_{4}^{k}\left(\sigma_{4}^{2}, d\right) .
\end{gathered}
$$

Проводя рассуждения, изложенные в лемме 2, легко показать, что система (9) эквивалентна системе

$$
\begin{gathered}
F_{2}^{-1} F_{1}^{-1} \sigma_{1}^{4 k} F_{1} F_{2}=\varphi_{1}^{k}\left(\sigma_{1}^{4}, \sigma_{2}^{4}\right), \quad F_{2}^{-1} F_{1}^{-1} \sigma_{2}^{4 k} F_{1} F_{2}=\varphi_{3}^{k}\left(\sigma_{1}^{4}, \sigma_{2}^{4}\right) ; \\
\left(F_{2}^{-1} F_{1}^{-1} \sigma_{1}^{-4 k} F_{1} F_{2}\right) F_{3}^{-1}\left(F_{2}^{-1} F_{1}^{-1} \sigma_{1}^{4 k} F_{1} F_{2}\right) F_{3}=1, \\
\left(F_{2}^{-1} F_{1}^{-1} \sigma_{2}^{-4 k} F_{1} F_{2}\right) F_{3}^{-1}\left(F_{2}^{-1} F_{1}^{-1} \sigma_{2}^{4 k} F_{1} F_{2}\right) F_{3}=1 ; \\
\left(F_{2}^{-1} F_{1}^{-1} \sigma_{1}^{-4 k} F_{1} F_{2}\right) F_{4}^{-1}\left(F_{2}^{-1} F_{1}^{-1} \sigma_{1}^{4 k} F_{1} F_{2}\right) F_{3}^{-1} \sigma_{4}^{2 k} F_{3} F_{4}=\varphi_{2}^{k}\left(\sigma_{4}^{2}, d\right), \\
\left(F_{2}^{-1} F_{1}^{-1} \sigma_{2}^{-4 k} F_{1} F_{2}\right) F_{4}^{-1}\left(F_{2}^{-1} F_{1}^{-1} \sigma_{2}^{4 k} F_{1} F_{2}\right) F_{3}^{-1} d^{k} F_{3} F_{4}=\varphi_{4}^{k}\left(\sigma_{4}^{2}, d\right) .
\end{gathered}
$$

Решения систем (10) и (11) удовлетворяют соответственно леммам 4 и 5 . Используя лемму 6 и переписывающий процесс $\tau$, второе равенство из (12) перепишем следующим образом:

$$
\tau\left(F_{2}^{-1} F_{1}^{-1} \sigma_{2}^{-4 k} F_{1} F_{2}\right) \tau^{-1}\left(F_{4}\right) \tau\left(F_{2}^{-1} F_{1}^{-1} \sigma_{2}^{4 k} F_{1} F_{2}\right) \tau^{k}(d) \tau\left(F_{4}\right)=\tau^{k}\left(\varphi_{4}\left(\sigma_{4}^{2}, d\right)\right)
$$

Используя равенство $\tau\left(F_{2}^{-1} F_{1}^{-1} \sigma_{2}^{4 k} F_{1} F_{2}\right)=\varphi_{3}^{k}\left(s_{1,2}^{2}, s_{2,3}^{2}\right)$, преобразуем данное соотношение к виду

$$
\varphi_{3}^{-k}\left(s_{1,2}^{2}, s_{2,3}^{2}\right) \tau^{-1}\left(F_{4}\right) \varphi_{3}^{k}\left(s_{1,2}^{2}, s_{2,3}^{2}\right) \tau^{k}(d) \tau\left(F_{4}\right)=\varphi_{4}^{k}\left(s_{4,5}, \tau(d)\right) .
$$

Пусть теперь

$$
\tau\left(F_{4}\right) \mp \prod_{i=1}^{n} Q_{i}\left(s_{4,5}, \tau(d)\right) P_{i}\left(s_{2,5}, s_{3,5}\right),
$$

где $Q_{1}\left(s_{4,5}, \tau(d)\right)$ и $P_{n}\left(s_{2,5}, s_{3,5}\right)$ могут быть равны 1 . 
Применяя соотношения (1) к произведению

$$
\begin{aligned}
& \varphi_{3}^{-k}\left(s_{1,2}^{2}, s_{2,3}^{2}\right) \tau^{-1}\left(F_{4}\right) \varphi_{3}^{k}\left(s_{1,2}^{2}, s_{2,3}^{2}\right) \\
& \quad \text { ㅍ } \varphi_{3}^{-k}\left(s_{1,2}^{2}, s_{2,3}^{2}\right)\left(\prod_{i=n}^{1} P_{i}^{-1}\left(s_{2,5}, s_{3,5}\right) Q_{i}^{-1}\left(s_{4,5}, \tau(d)\right)\right) \varphi_{3}^{k}\left(s_{1,2}^{2}, s_{2,3}^{2}\right),
\end{aligned}
$$

получаем

$$
\varphi_{3}^{-k}\left(s_{1,2}^{2}, s_{2,3}^{2}\right) \tau^{-1}\left(F_{4}\right) \varphi_{3}^{k}\left(s_{1,2}^{2}, s_{2,3}^{2}\right)=\prod_{i=n}^{1} P_{i}^{\prime-1}\left(s_{1,5}, s_{2,5}, s_{3,5}\right) Q_{i}^{-1}\left(s_{4,5}, \tau(d)\right)
$$

где

$$
P_{i}^{\prime-1}\left(s_{1,5}, s_{2,5}, s_{3,5}\right)=\varphi_{3}^{-k}\left(s_{1,2}^{2}, s_{2,3}^{2}\right) P_{i}^{-1}\left(s_{2,5}, s_{3,5}\right) \varphi_{3}^{k}\left(s_{1,2}^{2}, s_{2,3}^{2}\right), \quad i=1, \ldots, n .
$$

Элемент $s_{1,5}$ можно записать как $s_{1,5}=\tau(d) s_{4,5}^{-1} s_{3,5}^{-1} s_{2,5}^{-1}$. Поэтому

$$
P_{i}^{\prime-1}\left(s_{1,5}, s_{2,5}, s_{3,5}\right)=P_{i}^{\prime-1}\left(\tau(d) s_{4,5}^{-1} s_{3,5}^{-1} s_{2,5}^{-1}, s_{2,5} s_{3,5}\right), \quad i=1, \ldots, n .
$$

Представим теперь каждое $P_{i}^{\prime-1}$ в несократимой форме в свободных образующих $s_{4,5}, s_{3,5}, s_{2,5}, \tau(d)$ подгруппы $U_{4}$, т.е.

$$
\begin{aligned}
P_{i}^{\prime-1}\left(s_{1,5}, s_{2,5}, s_{3,5}\right)= & \left(\tau(d) s_{4,5}^{-1}\right)^{t_{i, 1}} P_{i, 1}^{\prime-1}\left(s_{2,5}, s_{3,5}\right)\left(\tau(d) s_{4,5}^{-1}\right)^{t_{i, 2}} \\
& \times P_{i, 2}^{\prime-1}\left(s_{2,5}, s_{3,5}\right) \cdots\left(\tau(d) s_{4,5}^{-1}\right)^{t_{i}, m_{i}} P_{i, m_{i}}^{\prime-1}\left(s_{2,5}, s_{3,5}\right),
\end{aligned}
$$

где $t_{i, 1}$ может быть равно 0, a $P_{i, m_{i}}^{\prime-1}$ - равно 1 .

Подставим полученное выражение (14) в левую часть (13). Имеем

$$
\begin{aligned}
& \left(\prod_{i=n}^{1}\left(\tau(d) s_{4,5}^{-1}\right)^{t_{i, 1}} P_{i, 1}^{\prime-1}\left(s_{2,5}, s_{3,5}\right)\left(\tau(d) s_{4,5}^{-1}\right)^{t_{i, 2}} P_{i, 2}^{\prime-1}\left(s_{2,5}, s_{3,5}\right) \cdots\left(\tau(d) s_{4,5}^{-1}\right)^{t_{i}, m_{i}}\right. \\
& \left.\quad \times P_{i, m_{i}}^{\prime-1}\left(s_{2,5}, s_{3,5}\right), Q_{i}^{-1}\left(s_{4,5}, \tau(d)\right)\right) \tau^{k}(d)\left(\prod_{i=n}^{1} Q_{i}\left(s_{4,5}, \tau(d)\right) P_{i}\left(s_{2,5}, s_{3,5}\right)\right) \\
& \quad=\varphi_{4}^{k}\left(s_{4,5}, \tau(d)\right)
\end{aligned}
$$

Пусть

$$
\begin{aligned}
& \prod_{i=n}^{1}\left(\tau(d) s_{4,5}^{-1}\right)^{t_{i, 1}} P_{i, 1}^{\prime-1}\left(s_{2,5}, s_{3,5}\right)\left(\tau(d) s_{4,5}^{-1}\right)^{t_{i, 2}} P_{i, 2}^{\prime-1}\left(s_{2,5}, s_{3,5}\right)\left(\tau(d) s_{4,5}^{-1}\right)^{t_{i}, m_{i}} \\
& \quad \times P_{i, m_{i}}^{\prime-1}\left(s_{2,5}, s_{3,5}\right), Q_{i}^{-1}\left(s_{4,5}, \tau(d)\right) \mp T, \\
& \prod_{i=1}^{n} Q_{i}\left(s_{4,5}, \tau(d)\right) P_{i}\left(s_{2,5}, s_{3,5}\right)=S .
\end{aligned}
$$

Тогда (15) примет вид $T \tau^{k}(d) D=\varphi_{4}^{k}\left(s_{4,5}, \tau(d)\right)$. 
Выполним сокращения в $T$ в свободной группе $\left\langle\tau(d), s_{2,5}, s_{3,5}, s_{4,5}\right\rangle$, причем сокращения между $Q_{i}$ и $\left(\tau(d) s_{4,5}^{-1}\right)^{t}$ будем вьполнять следующим образом. Если

$$
\left(Q_{i}\left(\tau(d) s_{4,5}^{-1}\right)^{t}\right)^{\eta}=\left(Q_{i}^{\prime} s_{4,5}^{-1}\left(\tau(d) s_{4,5}^{-1}\right)^{t^{\prime}}\right)^{\eta}
$$

где $\left|t^{\prime}\right|<|t|$ и $\eta= \pm 1$, либо

$$
\left(Q_{i}\left(\tau(d) s_{4,5}^{-1}\right)^{t}\right)^{\xi}=\left(Q_{i}^{\prime} \tau^{-1}(d)\left(\tau(d) s_{4,5}^{-1}\right)^{t^{\prime}}\right)^{\xi}
$$

где $\left|t^{\prime}\right|<|t|$ и $\xi= \pm 1$, то в первом случае к $Q_{i}^{\prime}$ присоединяем $s_{4,5}^{-1}$, во втором $-\tau^{-1}(d)$. При этом $\left|Q_{i}^{\prime}\right| \leqslant\left|Q_{i}\right|$. В результате вьполненных сокращений возможны следующие случаи:

1) $T=1$;

2) $T=T_{1} P\left(s_{2,5}, s_{3,5}\right)$;

3) $T=T_{1} P\left(s_{2,5}, s_{3,5}\right)\left(\tau(d) s_{4,5}^{-1}\right)^{p} Q^{\prime}\left(\tau(d), s_{4,5}\right)$;

4) $T=T_{1}\left(\tau(d) s_{4,5}^{-1}\right)^{p}, p \neq 0$.

В случае 1) (15) примет вид

$$
\tau^{k}(d)\left(\prod_{i=1}^{n} Q_{i}\left(s_{4,5}, \tau(d)\right) P_{i}\left(s_{2,5}, s_{3,5}\right)\right)=\varphi_{4}^{k}\left(s_{4,5}, \tau(d)\right) .
$$

Так как $\sum_{i=1}^{n}\left|Q_{i}\right|<2\left|F_{4}\right| \ll k$, в последнем равенстве $\tau^{k}(d)$ не может полностью сократиться; более того, это равенство рассматривается в свободной группе со свободными образующими $\tau(d), s_{2,5}, s_{3,5}, s_{4,5}$, поэтому для того чтобы оно было справедливо, все $P_{i}\left(s_{2,5}, s_{3,5}\right)$ должны быть равны 1.

Случай 2) аналогичен случаю 1$)$.

В случае 3) (15) имеет вид

$$
T_{1} P\left(s_{2,5}, s_{3,5}\right)\left(\tau(d) s_{4,5}^{-1}\right)^{p} Q^{\prime}\left(\tau(d), s_{4,5}\right) \tau^{k}(d) S=\varphi_{4}^{k}\left(s_{4,5}, \tau(d)\right) .
$$

Заметим, что из сказанного вьше $Q^{\prime} \leqslant \sum_{i=1}^{n}\left|Q_{i}\right| \leqslant\left|F_{4}\right| \ll k / 2$, поэтому при сокращении в произведении $Q^{\prime}\left(\tau(d), s_{4,5}\right) \tau^{k}(d) S$ останется какая-либо степень $\tau^{k^{\prime}}(d), k^{\prime}>1$, а при сокращении в произведении $\left(\tau(d) s_{4,5}^{-1}\right)^{p} \tau^{k^{\prime}}(d)$ может сократиться лиш один образующий $\tau^{\xi}(d), \xi= \pm 1$. Поэтому и в данном случае все $P_{i}\left(s_{2,5}, s_{3,5}\right)$ должны быть равны 1.

Случай 4) аналогичен случаю 3 ).

Из приведенных рассуждений следует доказательство леммы.

Лемма 9. Если имеет место (4)-(6), то существует коса $L_{2}\left(\sigma_{4}^{2}, d\right)$, удовлетворяющая системе

$$
L_{2}^{-1} \sigma_{4}^{2} L_{2}=\varphi_{2}\left(\sigma_{4}^{2}, d\right), \quad L_{2}^{-1} d L_{2}=\varphi_{4}\left(\sigma_{4}^{2}, d\right) .
$$

ДоКАЗАТЕЛЬСТво. Из леммы 5 следует, что существует коса $F_{3}$, являющаяся решением (5), такая, что $\tau\left(F_{3}\right)=\left(s_{1,4} s_{2,4} s_{3,4}\right)^{k}, k \in \mathbb{Z}$. По лемме $7 \tau^{-1}\left(F_{3}\right) s_{4,5} \tau\left(F_{3}\right)=$ $\tau^{k}(d) s_{4,5} \tau^{-k}(d)$ или в $B_{5} F_{3}^{-1} \sigma_{4}^{2} F_{3}=d^{k} \sigma_{4}^{2} d^{-k}$. По лемме $8 F_{4}=F_{4}\left(\sigma_{4}^{2}, d\right)$. Положим $L_{2}=d^{-k} F_{4}\left(\sigma_{4}^{2}, d\right)$. Тогда $L_{2}$ удовлетворяет системе (16).

Из лемм 4 и 9 следует доказательство теоремы. 
ОПРЕДЕЛЕНИЕ 1. В групп $G$ неразрешима проблема сопряженности подгрупп, если не существует алгоритма, позволяющего для любых двух ее конечно порожденных подгрупп установить, сопряжены ли они в $G$ или нет.

ОПРЕДЕЛЕНИЕ 2. В группе $G$ разрешима частная проблема сопряженности подәрynn, если существует алгоритм, позволяющий для любой ее конечно порожденной подгрупшы $H$ и фиксированной конечно порожденной подгруппы $H_{0}$ установить, сопряжены ли они в $G$ или нет.

ТЕОРема 3. В группе $R_{5}$ неразрешима проблема сопряэсенности подгрупп.

ДокАЗАтЕльство. Как указывалось вьше, $R_{5}$ содержит в качествеподгруппы прямое произведение $\left\langle\sigma_{1}^{4}, \sigma_{2}^{4}\right\rangle \times\left\langle\sigma_{4}^{2}, d\right\rangle$ двух свободных групп ранга 2 . Пусть

$$
\left\{a_{1}, a_{2} ; R_{1}\left(a_{1}, a_{2}\right), \ldots, R_{n}\left(a_{1}, a_{2}\right)\right\rangle
$$

- группа П. С. Новикова [6]. Возьмем в качестве фиксированной подгрупшы

$$
H_{1}=\left\langle\sigma_{1}^{4} \sigma_{2}^{4}, \sigma_{4}^{2} d, R_{1}\left(\sigma_{1}^{4}, \sigma_{2}^{4}\right), \ldots, R_{n}\left(\sigma_{1}^{4}, \sigma_{2}^{4}\right)\right\rangle,
$$

где $R_{i}\left(\sigma_{1}^{4}, \sigma_{2}^{4}\right), i=1, \ldots, n$, получены из определяющих слов групшы Новикова. В [7] доказано, что если в прямом произведении двух свободных групा ранга 2 разрешима частная проблема сопряженности подгруп, то разрешима частная проблема вхождения. А именно, для того чтобы определить, принадлежит ли произвольное слово $W$ группы $\left\langle\sigma_{1}^{4}, \sigma_{2}^{4}\right\rangle \times\left\langle\sigma_{4}^{2}, d\right\rangle$ фиксированной подгрупе $H_{1}$ или нет, достаточно знать, будут ли сопряжены подгрупшы $H_{1}$ и $\left\langle W, H_{1}\right\rangle$. Из [7] и [8] следует, что в $\left\langle\sigma_{1}^{4}, \sigma_{2}^{4}\right\rangle \times\left\langle\sigma_{4}^{2}, d\right\rangle$ неразрешима проблема сопряженности подгруп. Из этого факта и теоремы 2 следует утверждение теоремы 3 .

\section{СПИСОК ЦИТИРОВАННОЙ ЛИТЕРАТУРЫ}

[1] Burau W. Über Zopfinvarianten // Abh. Math. Sem. Univ. Hamburg. 1932. V. 9. P. 117-124.

[2] Artin E. Theory of braids // Ann. of Math. 1947. V. 48. P. 101-126.

[3] Марков А. А. Основы алгебраической теории кос // Тр. МИАН. 1945. Т. 16. С. 1-54.

[4] Добрынина И. В. О неразрешимости проблемы сопряженности подгрупп в группе крашеных кос $R_{n+1}(n \geqslant 5)$ // Алгоритмические проблемы теории групп и полугрупп. Тула: ТГПИ, 1994. C. $62-70$.

[5] Акименков А. М. О подгруппах группы кос B5 // Матем. заметки. 1991. Т. 50. №6. С. 3-13.

[6] Новиков П. С. Об алгоритмической неразрешимости проблемы тождества слов в теории групп // Тр. МИАН. 1955. Т. 44. С. 3-143.

[7] Безверхний В. Н. Неразрешимость проблемы сопряженности подгрупп для свободного произведения свободных групп с объединением // Сб. научн. тр. кафедры высшей математики. Вып. 3. Тула: ТГПИ, 1975. С. 90-94.

[8] Михайлова К.А. Проблема вхождения для прямых произведений групп // Матем. сб. 1966. T. 70. № 2. C. 241-251. 